\title{
RESTRICTIONS OF ANALYTIC FUNCTIONS. I
}

\author{
MARVIN ROSENBLUM AND JAMES ROVNYAK ${ }^{1}$
}

ABSTRACT. An operator theoretic method is used to characterize restrictions of boundary functions of $H^{2}$ and $H^{\infty}$ functions and to obtain a generalization of a theorem of Loewner.

1. Introduction. The problem of recapturing an analytic function from a restriction of its boundary function was treated in 1933 by Golusin and Krylov [2]. Elaborating on an idea of Carleman, these authors exhibited a sequence of approximants for the function, constructed from a restriction of the boundary function, which converges to the function under very general conditions. See also Priwalow [8]. For the case of an $H^{p}$ function on the unit disk, Patil [7] in 1972 constructed a sequence of approximants which is norm convergent, and he characterized the class of restrictions of boundary functions of $H^{p}$ functions as the class of functions for which the approximants are norm bounded. Loewner [6] in 1934 characterized a different class of functions with conditions of a different nature. To state Loewner's theorem, consider a function $w$ which is real valued and continuously differentiable on an interval $(a, b)$. Then for $w$ to be the restriction of a function $W$ which is holomorphic in the complex plane, slit along $(-\infty, a]$ and $[b,+\infty)$, and has a nonnegative imaginary part in the upper half-plane, it is necessary and sufficient that

$$
\sum_{j, k=1}^{n} \frac{w\left(x_{k}\right)-w\left(x_{j}\right)}{x_{k}-x_{j}} c_{j} c_{k}^{*} \geq 0
$$

for any finite set $x_{1}, \cdots, x_{n}$ of points in $(a, b)$ and complex numbers $c_{1}$, $\cdots, c_{n}$. The' after the summation symbol indicates that $\left[w\left(x_{k}\right)-w\left(x_{j}\right)\right] /\left(x_{k}-x_{j}\right)$ is to be replaced by $w^{\prime}(u)$ if $x_{k}=x_{j}=u$. For references to other treatments of Loewner's theorem and a refinement of the theorem, see our previous paper [10]. Other forms of the problem of recaptur-

Received by the editors January 8, 1974.

AMS (MOS) subject classifications (1970). Primary 30A78, 30A76; Secondary 47B35, 44A15.

Key words and phrases. Hardy classes, Toeplitz operators, Hilbert transform, functions with positive imaginary part.

${ }^{1}$ Research supported by NSF Grant GP-31483X. 
ing an analytic function from a restriction of its boundary function have been considered by Krein and Nudel'man [4] and van Winter [11].

In this paper we present an operator theoretic method which yields new characterizations of restrictions of $H^{2}$ and $H^{\infty}$ functions and a generalization of Loewner's theorem in which the interval $(a, b)$ is replaced by a Borel set. The conditions require the boundedness or nonnegativity of certain quadratic forms involving the finite Hilbert transform.

2. Prefatory comments. Notation introduced here will be used throughout the paper. Let $m$ denote Lebesgue measure on the real line $R$. Fix a Borel set $\Delta$ on $R$ such that neither $\Delta$ nor $R-\Delta$ are $m$-null sets. The Lebesgue spaces $L^{p}(R)$ and $L^{p}(\Delta)$ are defined with respect to the measures $m$ and $m \mid \Delta$, respectively $(1 \leq p \leq \infty)$. We write $\|\cdot\|_{p}$ for the norm in both spaces; $\langle\cdot, \cdot\rangle_{R}$ and $\langle\cdot, \cdot\rangle_{\Delta}$ denote the inner products in $L^{2}(R)$ and $L^{2}(\Delta)$, respectively. The identity relation "=a.e." for functions in Lebesgue spaces is written ".". The characteristic function $\chi_{\Delta}$ of $\Delta$ is used sym" bolically to indicate both projection and embedding: if, say, $f \in L^{2}(R)$, then $\chi_{\Delta} f$ is identified with the restriction of $f$ to $\Delta$, i.e. the projection of $f$ in $L^{2}(\Delta)$. On the other hand, if $f \in L^{2}(\Delta)$, then $\chi_{\Delta} f$ is often written for the function which is equal to $f$ on $\Delta$ and 0 on $R-\Delta$, i.e. the embedding of $f$ in $L^{2}(R)$.

The Hardy spaces $H^{p}(1 \leq p \leq \infty)$ may be viewed as closed subspaces of the Lebesgue spaces $L^{p}(R)$ or as the spaces of analytic functions $F(z)$, $y>0$, for which

$$
\|F\|_{p}= \begin{cases}\sup _{y>0}\left(\int_{-\infty}^{+\infty}|F(x+i y)|^{p} d x\right)^{1 / p}, & 1 \leq p<\infty, \\ \sup _{y>0}|F(x+i y)|, & p=\infty,\end{cases}
$$

is finite. The interpretation is usually forced by the context in which the spaces appear. For the case $p=2, P: L^{2}(R) \rightarrow H^{2}$ denotes the corresponding orthogonal projection or canonical mapping. When $H^{2}$ is viewed as a space of analytic functions defined in the upper half-plane, then for any $f \in L^{2}(R), F=P f$ is given by

$$
F(z)=\frac{1}{2 \pi i} \int_{-\infty}^{+\infty} \frac{f(t)}{t-z} d t, \quad y>0 .
$$

The Hilbert transform $\tau$ is the linear transformation on $L^{2}(R)$ defined by

$$
(\tau f)(x) \doteq P V \frac{1}{\pi} \int_{-\infty}^{+\infty} \frac{f(t)}{t-x} d t, \quad f \in L^{2}(R) .
$$


The finite Hilbert transform $\tau_{\Delta}$ is defined as the compression of $\tau$ to $L^{2}(\Delta)$ : $\tau_{\Delta}=\chi_{\Delta} \tau \chi_{\Delta}$. We have $\tau^{2}=-I$ and $P=(I-i \tau) / 2$ as operators on $L^{2}(R)$, and $\chi_{\Delta} P \chi_{\Delta}=\left(I-i \tau_{\Delta}\right) / 2$ as operators on $L^{2}(\Delta)$.

For any $g \in L^{\infty}(R)$ the Toeplitz operator $T(g)$ is defined on $H^{2}$ by

$$
T(g) u \doteq P g u, \quad u \in H^{2} .
$$

If $g$ is essentially real valued and not 0 a.e., then $T(g)$ is selfadjoint and $\operatorname{ker} T(g)=(0)[3]$.

3. Restrictions of $H^{2}$ functions. Fix functions $u$ and $v$ with $u \in L^{2}(\Delta)$, $v \in L^{\infty}(\Delta)$, and $v \geq 0$ a.e. We assume that $v$ is not 0 a.e. We consider the problem of giving conditions for the existence of a function $f \in H^{2}$ such that $u \doteq v f$ on $\Delta$.

Theorem 1. Let $u$ and $v$ be as above, and let $M$ be a nonnegative constant. Let $T=T\left(\chi_{\Delta} v\right)$. Then the following are equivalent:

(i) there exists $f \in H^{2}$ such that $u \doteq v f$ on $\Delta$ and $\|f\|_{2}=M$;

(ii) there exists $f \in H^{2}$ such that $T f \doteq P_{\chi_{\Delta}} u$ and $\|f\|_{2}=M$;

(iii) for all $\gamma \in L^{2}(\Delta)$,

$$
2\left|\langle u, \gamma\rangle_{\Delta}\right|^{2} \leq M^{2}\left\langle\left(I-i \tau_{\Delta}\right) v \gamma, v \gamma\right\rangle_{\Delta}
$$

and this assertion is not true if $M^{2}$ is replaced by any smaller constant.

Lemma 1 (Landesman [5]). Let $T$ be a bounded selfadjoint operator on a Hilbert space $\mathcal{H}$ with $\operatorname{ker} T=(0)$. Then a given vector $g$ belongs to the range of $T$ if and only if $M=\sup [|\langle g, \gamma\rangle| /\|T \gamma\|: \gamma \in \mathcal{H}, \gamma \neq 0]<\infty$. In this case $g=T f$, where $\|f\|=M$.

Proof of Theorem 1. Assume (i) holds. Then from $u \doteq v f$ on $\Delta$ we get $P \chi_{\Delta} u \doteq P \chi_{\Delta} v f \doteq T f$, so (ii) holds. Conversely, if (ii) holds, then $P\left(\chi_{\Delta} u-\chi_{\Delta} v f\right)=0$ so $\chi_{\Delta}(u-v f) \in L^{2}(R) \ominus H^{2}$. Since $\chi_{\Delta}(u-v f)$ vanishes on a set of positive measure, it must vanish a.e. on $R$. Hence $u \doteq v f$ on $\Delta$ and (i) and (ii) are equivalent.

Next we show that (ii) implies (iii). If (ii) holds, then $P_{\chi_{\Delta}} u$ is in the range of $T$, so by the lemma

$$
\left|\left\langle P \chi_{\Delta} u, \gamma\right\rangle_{R}\right| \leq M\left\|P \chi_{\Delta} v \gamma\right\|_{2}
$$

for all $\gamma \in H^{2}$, and the constant $M$ is best possible. The inequality can be rewritten

$$
\left|\left\langle\chi_{\Delta} u, \gamma\right\rangle_{R}\right|^{2} \leq M^{2}\left\langle\chi_{\Delta} P \chi_{\Delta} v \gamma, v \gamma\right\rangle_{R}
$$


or

$$
\left|\langle v, \gamma\rangle_{\Delta}\right|^{2} \leq M^{2}\left\langle 1 / 2\left(I-i \tau_{\Delta}\right) \chi_{\Delta} v \gamma, \chi_{\Delta} v \gamma\right\rangle_{\Delta}
$$

where $\gamma \in H^{2}$. But the set of functions $\chi_{\Delta} \gamma, \gamma \in H^{2}$, is dense in $L^{2}(\Delta)$, so (iii) follows. The steps in this argument are reversible, so in fact (ii) and (iii) are equivalent. The result follows.

Theorem 2. Let $w$ be any complex valued Borel measurable function on $\Delta$, and let $M$ be a nonnegative constant. Then there exists a function $f \in H^{2}$ such that $f \mid \Delta \doteq w$ on $\Delta$ and $\|f\|_{2}=M$ if and only if

$$
2\left|\int_{\Delta} w \gamma^{*} d m\right|^{2} \leq M^{2}\left\langle\left(I-i \tau_{\Delta}\right) \gamma, \gamma\right\rangle_{\Delta}
$$

for all $\gamma \in L^{2}(\Delta)$ such that $w \gamma^{*} \in L^{1}(\Delta)$, and in this statement $M^{2}$ cannot be replaced by any smaller constant.

Proof. Write $w=u / v$, where $u \in L^{2}(\Delta), v \in L^{\infty}(\Delta)$, and $v>0$ on $\Delta$. (We can choose $u=\alpha w /(1+|w|), v=\alpha /(1+|w|)$, where $\alpha \in L^{2}(\Delta) \cap L^{\infty}(\Delta)$ and $\alpha>0$ on $\Delta$.) We obtain the result in a routine way by applying Theorem 1 with $\Delta$ replaced by sets of the form $\Delta_{\epsilon}=\Delta \cap\{v>\epsilon\}$, where $\epsilon>0$ is small enough that $\Delta_{\epsilon}$ is not an $m$-null set.

Theorem 3. Let $w$ and $M$ be given as in Theorem 2. Then there exists a function $f \in H^{2}$ such that $(1 / f) \mid \Delta \doteq w$ on $\Delta$ and $\|f\|_{2}=M$ if and only if

$$
2\left|\int_{\Delta} \gamma d m\right|^{2} \leq M^{2}\left\langle\left(I-i \tau_{\Delta}\right) w^{*} \gamma, w^{*} \gamma\right\rangle_{\Delta}
$$

for all $\gamma \in L^{1}(\Delta)$ such that $w^{*} \gamma \in L^{2}(\Delta)$, and in this assertion $M^{2}$ cannot be replaced by any smaller constant.

Proof. The argument is similar to the proof of Theorem 2. The conclusion is easily obtained when $w$ vanishes on a set of positive measure. When $w$ is not zero on any set of positive measure, we can choose $u \epsilon$ $L^{2}(\Delta), v \in L^{\infty}(\Delta), v>0$ a.e. on $\Delta$, such that $v \doteq u w$ on $\Delta$. The result follows from Theorem 1 as in the proof of Theorem 2.

4. Restrictions of $H^{\infty}$ functions. Now consider functions $u, v \in L^{\infty}(\Delta)$ such that $v \geq 0$ a.e. on $\Delta$ and $v$ is not 0 a.e. We seek conditions which imply that $u \doteq v f$ on $\Delta$ for some $f \in H^{\infty}$.

Theorem 4. Let $u$ and $v$ be as just stated, and let $M$ be a nonnegative constant. The following are equivalent: 
(i) there exists $f \in H^{\infty}$ such that $u \doteq v f$ on $\Delta$ and $\|f\|_{\infty}=M$;

(ii) there exists a bounded operator $B$ on $H^{2}$ to $H^{2}$ such that $T\left(\chi_{\Delta} u\right)=T\left(\chi_{\Delta} v\right) B$ and $\|B\|=M$;

(iii) for all $y \in L^{2}(\Delta)$,

$$
\left\langle\left(I-i \tau_{\Delta}\right) u^{*} \gamma, u^{*} \gamma\right\rangle_{\Delta} \leq M^{2}\left\langle\left(I-i \tau_{\Delta}\right) v \gamma, v \gamma\right\rangle,
$$

and here $M^{2}$ cannot be replaced by any smaller constant.

Lemma 2. Let $A$ and $T$ be bounded operators on a Hilbert space $\mathcal{H}$, and let $\operatorname{ker} T=(0)$. Let $M$ be a nonnegative constant. Then there exists a bounded operator $B$ on $\mathcal{H}$ such that $A=T B$ and $\|B\|=M$ if and only if $A A^{*} \leq M^{2} T T^{*}$, and in this inequality $M^{2}$ cannot be replaced by any smaller constant.

Proof. See Douglas [1].

Proof of Theorem 4. If (i) holds then $T\left(\chi_{\Delta} u\right)=T\left(\chi_{\Delta} v f\right)=T\left(\chi_{\Delta} v\right) T(f)$, where $\|T(f)\|=M$; hence (ii) holds with $B=T(f)$.

Let (ii) hold. Then for every $\gamma \in H^{2}, P\left(\chi_{\lrcorner} u \gamma-\chi_{\Delta} v B \gamma\right) \doteq 0$. As in the proof of Theorem 1 it follows that $u-v(B \gamma / \gamma) \doteq 0$ on $\Delta$. Therefore $B y / \gamma=f$ is independent of $\gamma$. Choosing $\gamma \in H^{2}$ such that $1 / \gamma \in H^{\infty}$, we see that $f \in H^{2}$. For any $\beta \in H^{2}$,

$$
\int_{R}|f \beta|^{2} d m=\int_{R}|B \beta|^{2} d m \leq\|B\|^{2}\|\beta\|_{2}^{2} .
$$

Hence $f \in H^{\infty}$ and $\|f\|_{\infty}=\|B\|=M$. Therefore (i) holds.

Let (ii) hold again. By Lemma 2, $T\left(\chi_{\Delta} u\right) T\left(\chi_{\Delta} u\right)^{*} \leq M^{2} T\left(\chi_{\Delta} v\right) T\left(\chi_{\Delta} v\right)^{*}$, with the constant $M^{2}$ being best possible. The inequality can be rewritten as $\left\|P \chi_{\Delta} u^{*} \gamma\right\|_{2}^{2} \leq M^{2}\left\|P \chi_{\Delta} v \gamma\right\|_{2}^{2}, \gamma \in H^{2}$. Since $\chi_{\Delta} H^{2}$ is dense in $L^{2}(\Delta)$, the last inequality holds for all $\gamma \in L^{2}(\Delta)$. The left side is

$$
\left\|P \chi_{\Delta} u^{*} \gamma\right\|_{2}^{2}=\left\langle\chi_{\Delta} P \chi_{\Delta} u^{*} \gamma, u^{*} \gamma\right\rangle_{\Delta}=1 / 2\left\langle\left(I-i \tau_{\Delta}\right) u^{*} \gamma, u^{*} \gamma\right\rangle_{\Delta},
$$

and similarly for the right side. Thus (iii) follows. To show that conversely (iii) implies (ii) we reverse the steps in this argument. The theorem follows.

Theorem 5. Let $w$ be any complex valued Borel measurable function on $\Delta$, and let $M$ be a nonnegative constant. Then there exists an $f \in H^{\infty}$ such that $f \mid \Delta \doteq w$ on $\Delta$ and $\|f\|_{\infty}=M$ if and only if

$$
\left\langle\left(I-i \tau_{\Delta}\right) w^{*} \gamma, w^{*} \gamma\right\rangle_{\Delta} \leq M^{2}\left\langle\left(I-i \tau_{\Delta}\right) \gamma, \gamma\right\rangle_{\Delta}
$$

for all $\gamma \in L^{2}(\Delta)$ such that $w^{*} \gamma \in L^{2}(\Delta)$, and $M^{2}$ cannot be replaced by any smaller constant. 
Theorem 6. Let $w$ and $M$ be given as in Theorem 5. Then there exists an $f \in H^{\infty}$ such that $(1 / f) \mid \Delta \doteq w$ on $\Delta$ and $\|f\|_{\infty}=M$ if and only if

$$
\left\langle\left(I-i \tau_{\Delta}\right) \gamma, \gamma\right\rangle_{\Delta} \leq M^{2}\left\langle\left(I-i r_{\Delta}\right) w^{*} \gamma, w^{*} \gamma\right\rangle_{\Delta}
$$

for all $\gamma \in L^{2}(\Delta)$ such that $w^{*} \gamma \in L^{2}(\Delta)$, and $M^{2}$ cannot be replaced by any smaller constant.

Proofs of Theorems 5 and 6. The arguments are similar to the proofs of Theorems 2 and 3; now we write $w$ as quotients of $L^{\infty}(\Delta)$ functions, and, of course, we use Theorem 4 instead of Theorem 1.

5. Generalization of Loewner's theorem. We now apply Cayley transforms in the previous results.

Theorem 7. Let $w$ be a complex valued Borel measurable function on $\Delta$ such that $\operatorname{Im} w(x) \geq 0$ a.e. on $\Delta$. Then for the existence of a function $W(z)$ holomorphic and having nonnegative imaginary part in the upper half plane such that $W(x+i 0)=w(x)$ a.e. on $\Delta$, it is necessary and sufficient that

$$
i\left\langle\left(w-w^{*}\right) \gamma, \gamma\right\rangle_{\Delta} \leq\left\langle\tau_{\Delta} w^{*} \gamma, \gamma\right\rangle_{\Delta}-\left\langle\tau_{\Delta} \gamma, w^{*} \gamma\right\rangle_{\Delta}
$$

for all $\gamma \in L^{2}(\Delta)$ such that $w^{*} \gamma \in L^{2}(\Delta)$.

Proof. Suppose $W(z)$ exists, and set $F(z)=[W(z)-i] /[W(z)+i], y>$ 0 . Let $f(x)=F(x+i 0)$ a.e. on $R$. Then $f \in H^{\infty}$ and $\|f\|_{\infty} \leq 1$. Also $f(x) \doteq$ $[w(x)-i] /[w(x)+i]$ on $\Delta$. By Theorem 5 ,

$$
\left\langle\left(I-i \tau_{\Delta}\right) \alpha\left(w^{*}+i\right) /\left(w^{*}-i\right), \alpha\left(w^{*}+i\right) /\left(w^{*}-i\right)\right\rangle_{\Delta} \leq\left\langle\left(I-i \tau_{\Delta}\right) \alpha, \alpha\right\rangle_{\Delta}
$$

for all $\alpha \in L^{2}(\Delta)$. Take $\alpha=\left(w^{*}-i\right) \gamma$, where $\gamma$ is any element of $L^{2}(\Delta)$ such that $w^{*} \gamma \in L^{2}(\Delta)$. The necessity of the condition follows. It is easy to reverse the steps in this argument to prove the sufficiency of the condition.

The generalization of Loewner's theorem now follows.

Theorem 8. Let $w$ be a real valued Borel measurable function on $\Delta$. For the existence of a function $W$ separately holomorphic for $y>0$ and $y<0$, having a nonnegative imaginary part for $y>0$ and satisfying $W\left(z^{*}\right)=$ $W(z)^{*}$ for $y \neq 0$, such that $w(x) \doteq W(x+i 0) \doteq W(x-i 0)$ on $\Delta$, it is necessary and sufficient that

$$
\left\langle\tau_{\Delta} w \gamma, \gamma\right\rangle-\left\langle\tau_{\Delta} \gamma, w \gamma\right\rangle \geq 0
$$

for all $\gamma \in L^{2}(\Delta)$ such that $w \gamma \in L^{2}(\Delta)$. 
Proof. Apply Theorem 7 and define $W(z)$ for $y<0$ by $W(z)=W\left(z^{*}\right)^{*}$.

To see that Theorem 8 implies Loewner's theorem, two things must be checked. One is that for a real valued continuously differentiable function $w(x)$ on an interval $\Delta=(a, b)$, conditions (1) and (2) are equivalent. It is easy to see that (1) is equivalent to the condition

$$
\int_{a}^{b} \int_{a}^{b} \frac{w(x)-w(t)}{x-t} f(t) f(x)^{*} d t d x \geq 0
$$

where $f(x)$ is an arbitrary continuous function with compact support on $(a, b)$, and (2) is equivalent to

$$
\lim _{\epsilon>0} \int_{a}^{b} \int_{a}^{b} \chi\{|x-t|>\epsilon\}(x, t) \frac{w(x)-w(t)}{x-t} f(t) f(x)^{*} d t d x \geq 0
$$

for the same class of functions. The equivalence of the last two conditions is clear. The other thing that we have to check is that the function $W(z)$ produced by Theorem 8 is analytic across $(a, b)$. Since $w(x)$ is bounded on closed subintervals of $(a, b)$, this follows, for example, from [9, Theorem 2.8, part (ii)], with $u=v=1$ in that theorem.

\section{REFERENCES}

1. R. G. Douglas, On majorization, factorization, and range inclusion of operators on Hilbert space, Proc. Amer. Math. Soc. 17 (1966), 413-415. MR 34 \#3315.

2. G. M. Golusin and W. J. Krylov, Generalization of a formula of Carleman and its application to analytic continuation, Mat. Sb. 40 (1933), 144-149.

3. Philip Hartman and Aurel Wintner, The spectra of Toeplitz's matrices, Amer. J. Math. 76 (1954), 867-882. MR 17, 499.

4. M. G. Krein and P. Ja. Nudel' man, On some new problems for Hardy class functions with continuous families of functions with double orthogonality, Dokl. Akad. Nauk SSSR 209 (1973), 537-540 = Soviet Math. Dokl. 14 (1973), 435-439.

5. E. M. Landesman, Hilbert space methods in elliptic partial differential equations, Pacific J. Math. 21 (1967), 113-131. MR 35 \#806.

6. K. Loewner, Über monotone matrix Funktionen, Math. Z. 38 (1934), 177216.

7. D. J. Patil, Representation of $H^{p}$-functions, Bull. Amer. Math. Soc. 78 (1972), 617-620. MR $45 \# 7069$.

8. I. I. Priwalow, Randeigenschaften analytischer Funktionen, Hochschilbücher für Mathematik, Band 25, VEB Deutscher Verlag der Wissenschaften, Berlin, 1956. MR 18, 727.

9. M. Rosenblum and J. Rovnyak, The factorization problem for nonnegative operator valued functions, Bull. Amer. Math. Soc. 77 (1971), 287-318. MR 42 \#8315.

10. - Two theorems on finite Hilbert transforms, J. Math. Anal. Appl. (to appear).

11. C. van Winter, Fredholm equations on a Hilbert space of analytic functions, Trans. Amer. Math. Soc. 162 (1971), 103-139. 